\title{
Evolution process of fine fraction of condensed combustion products of aluminized condensed systems: experimental study
}

\author{
Valery A. Babuk ${ }^{1, *}$, Nikita L. Budnyi ${ }^{1}$ \\ ${ }^{1}$ Baltic state technical university "VOENMEH", 190005 Saint Petersburg, Russia
}

\begin{abstract}
The experimental technique and the results of experimental study of the fine fraction of condensed combustion products (CCP) of aluminized condensed systems during their evolution are presented. An analysis was conducted and the interpretation of experimental data is given. The physical picture of formation and evolution of the fine fraction of CCP is proposed.
\end{abstract}

\section{Introduction}

Aluminized condensed systems (CS) are widely used in different power and propulsion systems nowadays. The formation of condensed combustion products (CCP) near the burning surface is an important feature of the burning process of these systems [1]. Condensed combustion products take part in a set of physical and chemical transformations during motion in a combustion products flow (called "evolution process" [2-3]) and, as a result, final CCP are formed. Condensed combustion products of aluminized CS consist of two fractions: the coarse - agglomerates, and the fine - smoke oxide particles (SOP) [1]. Agglomerates are products of a coalescence of condensed substances in a surface layer of a burning CS: they have relatively big size (from tens to hundreds and thousands of micrometers) and consist of aluminum and its oxide. Smoke oxide particles are fine $\mathrm{Al}_{2} \mathrm{O}_{3}$ particles (from tens of nanometers to tens of micrometers). In this work the results of the experimental investigation of CCP fine fraction evolution gathered in BSTU "VOENMEH" are presented and discussed.

\section{Experimental study}

\subsection{Experimental technique}

During experimental study, the CS specimens were burnt in the constant volume bomb (CVB) (fig. 1A) with the following collection and analysis of the CCP [2].

\footnotetext{
* Corresponding author: babuk@peterlink.ru
} 

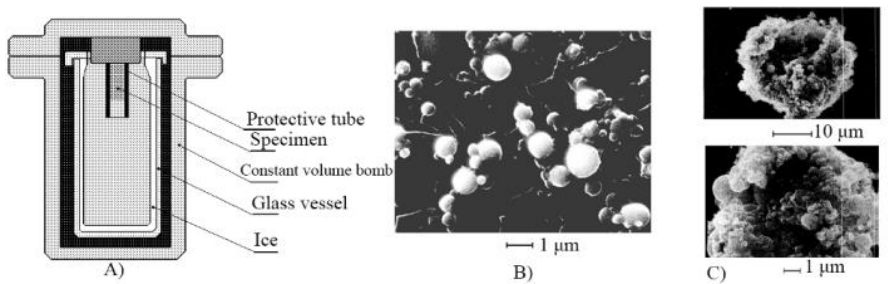

Fig. 1. A) Experimental set-up; B) Individual SOP; C) Coagulated SOP.

The constant volume bomb was pressurized with the inert gas. The glass vessel with a layer of ice on its inner surface was placed inside the CVB. The time of staying of the CCP in a flow of the combustion products was set through placing the specimen in the protective tubes of various lengths. This technique provides a possibility to analyze CCP at various distances from the burning surface. The constant volume bomb was sealed for at least 2 hours after combustion to provide a deposition of small particles. The fractions were separated using sieves. The individual SOP are shown on fig. 1B. The characteristics of dispersity were obtained during analysis of the whole totality of collected SOP using microanalyzers "COULTER 4" and Horiba LA-950. The collected SOP undergo an ultrasonic processing with an addition of sodium acid pyrophosphate for destruction of undesirable coagulants (рис.1C). The special technique was developed for determination of the optimal ultrasonic processing duration, which provides a destruction of the coagulants and prevents formation of new ones [1]. The chemical analysis and the qualitative analysis using microscope visualization also were conducted. The "ice tubes" (made of water and hydrogen peroxide) were using for the reduction of coagulation due to decomposition of protective tubes material (plexiglass and polytetrafluoroethylene) in the case of using extended tubes. The "ice tubes" were placed at the inner surface of protective tubes. The chemical analysis allowed to make a conclusion about almost complete absence of any substances besides $\mathrm{Al}_{2} \mathrm{O}_{3}$ in SOP.

The following SOP characteristics were studied: SOP mass share in CCP, presented by the fraction of initial metal in the propellant used to form SOP $Z_{\mathrm{m}}{ }^{\text {SOP }}$, and parameters of dispersity - the mass distribution density function $f_{\mathrm{m}}(d)$ and the mass-mean diameter $d_{43}$.

\subsection{Experimental results}

Table 1 presents the results of experimental study of SOP evolution for the ammonium perchlorate-based formulation N1-N5 [1], CSP-X4 [4], and for the ammonium dinitramidebased formulation CSP-X1 [4]. Figure 2 shows some examples of $f_{\mathrm{m}}(d)$ for various formulations at various distances from a CS surface.

The dispersion analysis of the collected particles allowed to make important conclusions:

- As a rule, SOP grow during evolution $-d_{43}$ increases during moving away from the surface. This phenomenon was observed for various formulations and pressure levels.

- SOP growth intensity can be various and depends on CS formulation, combustion conditions, agglomerates characteristics.

- Mass function of SOP size distribution density can be similar to unimodal or bimodal distribution, but there is a tendency to merging of modes or to disappearing of mode during evolution.

- SOP can reach large diameter (up to several microns).

- Fraction of initial metal in the propellant used to form SOP $Z_{\mathrm{m}}{ }^{\text {SOP }}$ increases during moving away from surface. 
Table 1. Experimental results on SOP dispersity during evolution.

\begin{tabular}{|c|c|c|c|}
\hline Formulation & Pressure, $\mathrm{MPa}$ & $\begin{array}{l}\text { Distance from CS } \\
\text { surface, } \mathrm{mm}\end{array}$ & $d_{43}, \mu \mathrm{m}$ \\
\hline \multirow{4}{*}{ N1 } & \multirow{2}{*}{1} & $*$ & $0.35 \pm 0.07$ \\
\hline & & 70 & $0.535 \pm 0.09$ \\
\hline & \multirow{2}{*}{6} & $*$ & $0.40 \pm 0.08$ \\
\hline & & 70 & $0.42 \pm 0.09$ \\
\hline \multirow{3}{*}{ N4 } & \multirow{3}{*}{6} & * & $0.55 \pm 0.05$ \\
\hline & & 30 & $0.62 \pm 0.05$ \\
\hline & & 70 & $0.67 \pm 0.06$ \\
\hline \multirow{2}{*}{ N5 } & \multirow{2}{*}{6} & $*$ & $0.80 \pm 0.16$ \\
\hline & & 70 & $1.35 \pm 0.20$ \\
\hline \multirow{3}{*}{ CSP-X1 } & \multirow{3}{*}{4} & $*$ & $0.52 \pm 0.05$ \\
\hline & & 30 & $0.61 \pm 0.06$ \\
\hline & & 70 & $0.68 \pm 0.07$ \\
\hline \multirow{3}{*}{ CSP-X4 } & \multirow{3}{*}{4} & $*$ & $0.54 \pm 0.05$ \\
\hline & & 30 & $0.63 \pm 0.06$ \\
\hline & & 70 & $0.70 \pm 0.07$ \\
\hline
\end{tabular}

* - combustion without protective tube

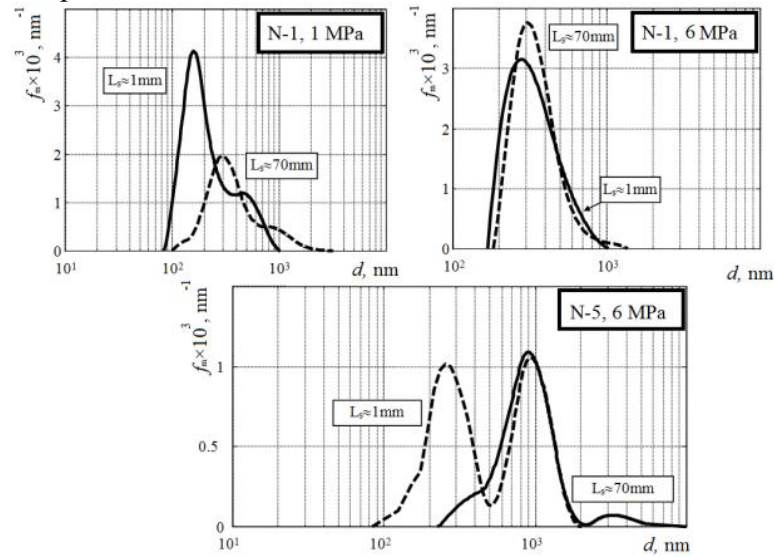

Fig. 2. Smoke oxide particles $f_{\mathrm{m}}(d)$ for the ammonium perchlorate-based CS on various distances from surface $\left(L_{\mathrm{s}}-\right.$ distance from CS surface).

\section{Interpretation of results: physical picture of SOP formation and evolution}

The common scheme of SOP formation and evolution is offered based on the analysis of experimental data and references on burning of aluminum particles in active environments (fig.3). 


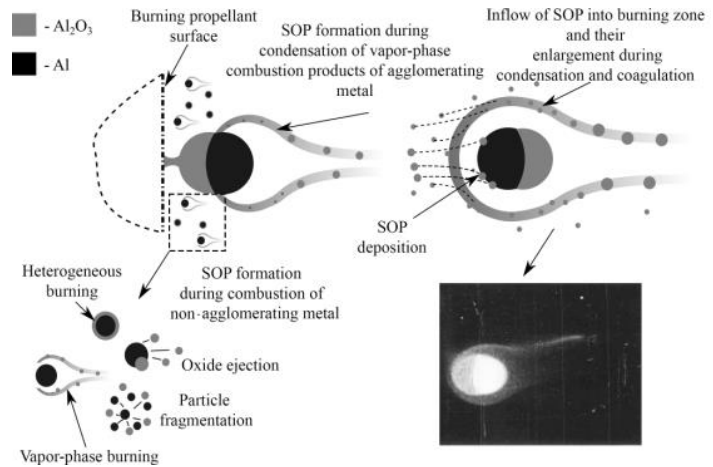

Fig. 3. Scheme of SOP formation and evolution.

The smoke oxide particles formation at the burning surface of $\mathrm{CS}$ is a result of combustion of non-agglomerating particle and burning of agglomerating particles. According to the common hypothesis, bimodal SOP distribution is a result of competition of oxide formation during vapor-phase burning and oxide accumulation on surface of particles. Word "mode" has to be understood conditionally - every "mode" in fact can be "polymodal". Apparently, the finest oxide particles (first mode) are formed during condensation of vapor-phase combustion products of non-agglomerating metal particles. The second mode, apparently, are formed during oxide accumulation on surfaces of nonagglomerating metal particles as a result of heterogeneous burning and, probably, diffusion and surface condensation of combustion products from zone of vapor-phase burning. The structure and characteristic particle sizes of the second mode are determined by dynamics of the accumulated oxide dispergation (or its absence). After oxide melting and starting of metal evaporation, there are some possible "scenarios": oxide ejection from particle surface (one or several droplets), "explosive" fragmentation of particle, transformation of oxide film into oxide "cap" without fragmentation or ejection. The final part of process is a complete combustion of residual metal core and formation of oxide particle as a result. The vapor-phase burning of agglomerating particles and the heterogeneous condensation of combustion products also can make a "contribution" to the second mode. The carbon particles (soot), which are formed during CS combustion, can act as nuclei in the heterogeneous condensation process [5]. Probably, the condensation behavior (homogeneous or heterogeneous) is determined by a concentration and size of nuclei in gas phase ("extraneous" particles, ions, impurities etc.) and also by size of burning particles. In other words, the condensation mechanism is determined by probability of presence of "extraneous" condensation centers in zone of vapor-phase burning.

The gas phase and the CCP are transformed during combustion products evolution [2, 3]. The essential phenomenon of the combustion products evolution is an agglomerate metal burning. Smoke oxide particles evolution is connected with this phenomenon process of metal vapor-phase burning is a source of aluminum sub-oxides which condense into $\mathrm{Al}_{2} \mathrm{O}_{3}$. In [6] SOP growth during evolution explained in the following way: during burning of agglomerate metal sub-oxides are being produced; SOP, which are in the velocity equilibrium with gas, can flow into agglomerates burning zones due to their velocity lag, and then SOP can act as nuclei (condensation centers). Sub-oxides in agglomerate burning zone condense into $\mathrm{Al}_{2} \mathrm{O}_{3}$ on existed SOP surfaces that resulting in a SOP growth. Particles coagulation is also possible. Growth rate during evolution depends on many factors: gas velocity, SOP concentration and size, agglomerates concentration and size, their velocity lag relative to gas, and combustion environment properties, which have an influence on CS and agglomerates combustion dynamics. 
The smoke oxide particles deposition on agglomerates can cause additional transformation of SOP concentration and dispersity. Deposition intensity increases with increasing of an agglomerate velocity lag and their concentration.

In a combustion chamber SOP growth can be probably more intensive due to effects of a turbulent flow leading to coagulation.

\section{Conclusions}

The experimental results on SOP evolution in combustion products flow are presented. It is assumed that the observed SOP growth is caused by inflow of SOP into the burning zones of agglomerates where particles grow during condensation of aluminum sub-oxides on their surfaces.

Authors are grateful to M. S. Malakhov and P. A. Naslednikov, who made a significant work in the development of experimental technique and experimentation in different years.

\section{References}

1. V.A. Babuk, V.A. Vasilyev, M.S. Malakhov, J. Propul. Power. 15, 783 (1999)

2. V.A. Babuk, V.A. Vasilyev, P.A. Naslednikov, Combustion of Energetic Materials (Begell House, New York, 2002)

3. V.A. Babuk, V.A. Vasilyev, J. Propul. Power. 18, 814 (2002)

4. V.A. Babuk, V.A. Vassiliev, V.V. Sviridov, Combust. Sci. Technol. 163, 261 (2001)

5. V.A. Babuk, N.L. Bydnyi, Khimicheskaya fizika i mezoskopiya, 17, 39 (2015) [In Russian]

6. V.A. Babuk, N.L. Bydnyi, Khimicheskaya fizika i mezoskopiya, 19, 5 (2017) [In Russian] 